\title{
Research on Modelica Based Modeling and Simulation of PCB AOI Imaging System
}

\author{
Jian Zhang ${ }^{1, a^{*}}$, Xiao Bo Peng ${ }^{2, b}$ \\ ${ }^{1}$ College of Mechantronics and Control Engineering, Shenzhen University, NO.3688,Nanhai Road, \\ Nanshan District, Shenzhen, China \\ ${ }^{2}$ College of Mechantronics and Control Engineering, Shenzhen University, NO.3688,Nanhai Road, \\ Nanshan District, Shenzhen, China \\ ajianzhang0312@foxmail.com, bengxb@szu.edu.cn
}

Keywords: Modelica; PCB; AOI; Imaging system; Modeling and simulation.

\begin{abstract}
The imaging system of PCB AOI is a complex electromechanical system which is coupled with mechanical, electronic, optical and control fields. The methods of modeling and simulation based on the unified modeling language Modelica can directly take the impact caused by multi-field coupling into consideration, while the traditional single field simulation methods can't. By the modeling and simulation methods based on Modelica, at first the basic models of PCB AOI imaging system in mechanical, optics and control fields are established. Then the imaging system model of PCB AOI is established according to the relation of the components' parameters. Finally, the sharpness of the image, an image quality evaluation parameter, is used for the evaluation of the PCB AOI imaging system. This method can not only be used to optimize the existing PCB AOI imaging system, but also be used to provide a platform for the research and development of new PCB AOI systems.
\end{abstract}

\section{Introduction}

Automatic optical inspection (AOI) has been used widely in the defect detection of PCB ${ }^{[1,2]}$. However, the domestic research on the AOI technology started relatively late. The domestic AOI equipment has been fallen behind in defect detection efficiency, accuracy, resolution and other aspects with foreign products. And it can't completely meet the actual needs of PCB production ${ }^{[3]}$. The PCB AOI imaging system provides the image for PCB defect detection system. The quality of the image determines directly the difficulty of defect detection algorithm of PCB and may affect the defect detection results. Therefore, the research on the modeling and simulation of PCB AOI imaging system can improve the performance level of the entire PCB AOI equipment timely.

PCB AOI imaging system is a complex electromechanical system coupled with mechanical, optical, control and other fields. The traditional modeling and simulation method that integrating after modeling in single field has a poor applicability to it because of the error caused during the fields combination. Modelica is an object-oriented and multi-field non-causal equation based modeling language. The seamless integration and data exchange can be achieved on the unified multi-domain modeling and simulation platform based on Modelica ${ }^{[4]}$.

Combined with the existing AOI project in this laboratory, this paper makes an introduction of the principle of linear CCD imaging at first. Then, on the basis of the research on the imaging process of PCB AOI system, it makes a detailed description of the modeling methods of PCB AOI imaging system based on unified modeling language Modelica. At last, it provides the modeling methods with a simulation and verification by the result of the sharpness of the image. 


\section{The imaging principle of linear CCD}

$\mathrm{CCD}$, made of the phototransistors arranged in the form of a matrix, is a type of electronic device converting the optical signals into electrical signals. The working process of CCD can be divided into two periods, the light integrator process and the charge transfer process. The main task of the light integration process is to generate and store the optical charge. In charge transfer process, the charge is transferred to the shift register to be output to the image acquisition card. The amount of charge is proportional to the light illumination and the optical integration time ${ }^{[5]}$. There is only one row of pixels generated during the linear CCD work cycle. As a result, for the linear CCD imaging system of PCB AOI, the complete image of PCB is finished by collecting and splicing the charge signals row by row.

\section{Lighting model}

The optical path of the PCB AOI imaging system studied is shown as the Fig. 1. The LED light source in a combination form sets up a stable field of light. The light is irradiated to the surface of the PCB and reflected. Then it passes through the lens and focuses on the linear CCD capture surface. The entire CCD imaging system will be sheltered while the CCD is scanning the PCB in order to ignore the more complex compact of the external ambient light. Therefore the Phong lighting model is adapt to describe the inside imaging system.

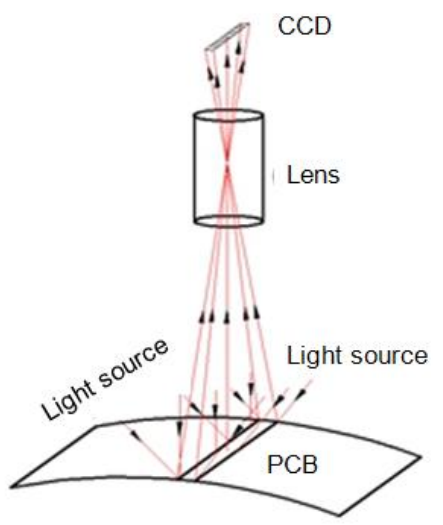

Figure.1 Optical imaging system schematic of the PCB AOI

Phong illumination model can be expressed as Eq. 1: the surface light intensity of a point to the viewpoint is the sum of the ambient reflected light intensity $I_{e}$, the ideal diffuse light intensity $I_{d}$ and the specular reflection light intensity $I_{s}{ }^{[6]}$.

$$
I=I_{e}+I_{d}+I_{s}
$$

\section{Modeling of imaging system}

According to the light propagation of the linear CCD imaging system, its modeling work includes five parts: standard image generation, PCB surface reflection, lens transmission imaging, mechanical motion system and linear array CCD scanning \& imaging system.

Standard image generation. The simulation model of imaging system takes the PCB standard bitmap as the input, while the actual PCB AOI system takes the PCB object. The PCB standard bitmap is transferred from PCB Gerber file (the standard format for the design and manufacture of circuit boards) which describes two-dimensional vector graphics. The data of the PCB Gerber file includes wiring layer and drilling layer. The wiring layer is used to describe the wiring line on the PCB substrate, while the drilling layer is used to describe the drilling hole. 
MWorks software is a modeling and simulation tool based on Modelica language, which supports both the text language modeling and the graphic modeling ${ }^{[7]}$. Therefore, the code information of Gerber files can be read by MWorks and used to draw the bitmap file according to the meaning of each code. As a result, the pixel gray value matrix of PCB standard image can be acquired.

Modeling of PCB surface reflection. The reflection phenomenon is mainly related to the light source, the surface of the irradiated object and the direction of the light. The field of view in the linear CCD imaging system is almost similar to the shape of the line. So the light intensity can be taken as the same value for the LED combination light source. That is to say, the light intensity value of the field of view is approximately independent of the spatial distribution.

The AOI system studied is used for the defect detection of PCB bare board, on which there are only copper and the substrate surface. PCB AOI equipment studied is used in red light source. The specular reflectance of copper in red light approaches 95\%. The impact of the light diffuse can be negligible. Therefore, the intensity of the light reflected from the copper of the PCB surface $I_{s}$ can be calculated as Eq. 2.The intensity of the light reflected from the substrate of the PCB surface $I_{d}$ can be calculated as Eq. 3.

$$
I_{s}=I_{p} K_{s}
$$

$$
I_{d}=I_{p} K_{d}
$$

$I_{p}$ represents the intensity of the light source; $K_{s}$ represents specular reflectance; $K_{d}$ represents diffuse reflectance.

Modeling of lens transmission process. The main performance parameters of the lens are focal length, working distance and aperture. Fig. 2 shows the correspondence between the parameters of the lens. WD represents the working distance; $f$ represents the focal length; FOV represents for the field of view; $V$ and $H$ represent the of length and the width ${ }^{[8]}$.

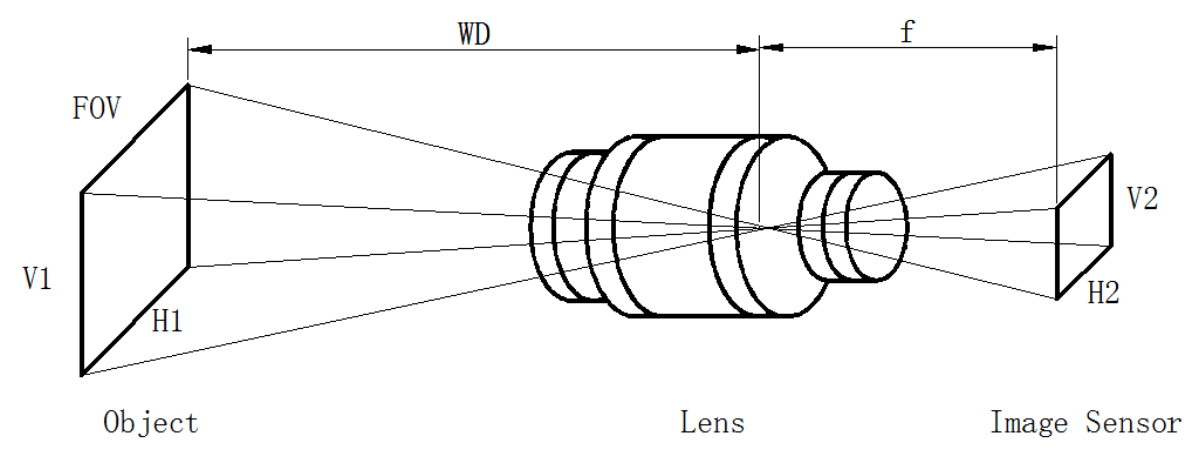

Figure. 2 Correspondence between the parameters of the lens

According to the figure, the area of the field of view corresponding to a single CCD pixel Area can be calculated as Eq. 4 .

$$
\text { Area }=H_{1} \cdot V_{1}=H_{2} \cdot V_{2}\left(\frac{W D}{f}\right)^{2}
$$

In the imaging system, the unit size of the image is determined to the size of the CCD pixel; the focal length of the prime lens is fixed; and the working distance is determined by the distance between the lens and the PCB in the mechanical motion system of PCB AOI. 
The light exposure can be adjusted by the lens aperture. The aperture values of the prime lens have been standardized. Under the same condition, the amount of light exposure will be halved as the size of the aperture progressively increases.

While establishing the model of lens based on Modelica, the characteristics of the lens imaging can be described by the ratio between the focal length and the working distance. Furthermore, according to the maximum aperture value of the lens, the aperture values available are determined. Different exposure scale factors can be set for different aperture value according to the application characteristics of the aperture.

Modeling of mechanical motion system. The mechanical motion system of PCB AOI not only reflects the distance between the lens and the PCB object, but also reflects the positional relationship between the CCD and PCB when the CCD imaging system works.

In the PCB AOI developed in this laboratory, PCB is fixed on the workbench, which can be linearly reciprocated in the $\mathrm{X}$-axis direction. Light source, $\mathrm{CCD}$ and camera are assembled on a component which can be linearly reciprocated in the Y-axis direction. Therefor the entire area of PCB can be scanned by linear CCD row-by-row. CCD and lens can be linearly reciprocated in the Z-axis direction in order to focus the imaging system. Lead screw drive is used for the motion of each axis. The final mechanism motion model established in MWorks is shown in Fig. $3^{[9]}$.

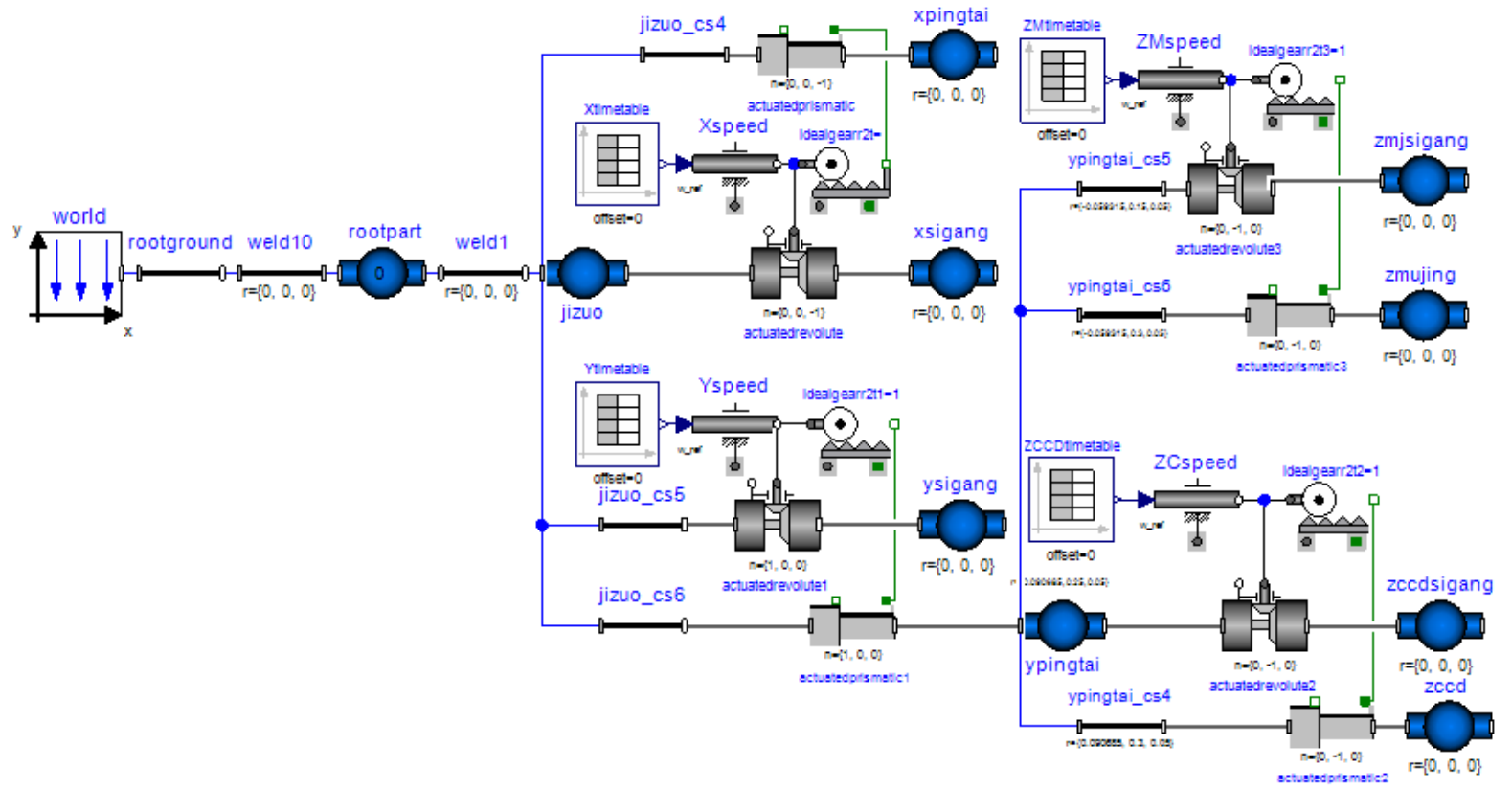

Figure. 3 Simulation model of AOI mechanical motion system

Modeling of linear array CCD scanning \& imaging system. During each exposure time, the value of optical signal for each CCD pixel is proportional to the light intensity, the imaging area and the integration time. So it can be calculated as Eq. 5.

$$
g(x, y)=\int_{0}^{T} \int_{x-a / 2}^{x+a / 2} \int_{y-b / 2}^{y+b / 2} h\left(x^{\prime}, y^{\prime}\right) d x^{\prime} d y^{\prime} d t
$$

$g(x, y)$ represents the value of optical signal collected from PCB, $h\left(x^{\prime}, y^{\prime}\right)$ indicates the light intensity, $T$ represents the cycle time of integration, $a$ and $b$ denote the length and width of the imaging cell ${ }^{[10]}$.

During the linear array CCD scanning \& imaging system modeling based on Modelica, the value of light intensity can be approximated to be independent of time for the reason that the light source illumination is almost stable. Each area of field can be divided into $n$ pieces according to the value of 
the light intensity. Suppose that the light intensity of the area $i$ is $I_{i}$ and the area is $A_{i}$, then the Eq. 5 changes into Eq. 6

$$
g(x, y)=T \sum_{i=1}^{n} A_{i} I_{i}
$$

Then the optical signal value of each pixel during the once scanning course can be obtained. And finally the entire PCB image can be formed by collecting and splicing the pixel values of all the scanning courses. The image quality evaluation parameters received by calculating the pixel values of the image can be used for the entire imaging system AOI evaluation.

\section{Simulation and verification}

Taking these various processes in the linear CCD scanning and imaging together, a linear CCD imaging model of PCB AOI system is established as shown in Fig. 4.

Take a standard PCB image as the input of the linear CCD imaging model. Referring the specific parameters of PCB AOI elements in this laboratory, the sharpness of the PCB scanning image is shown in Fig. 5.

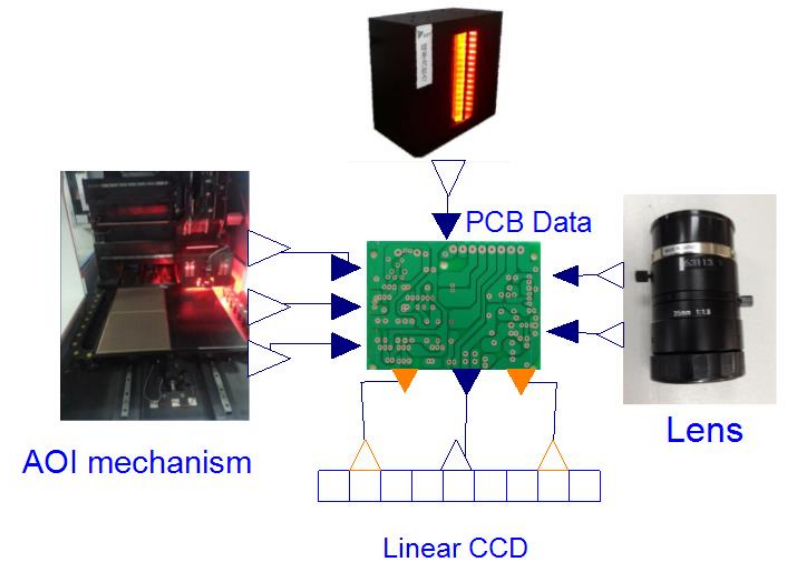

Figure. 4 Simulation model of the AOI system

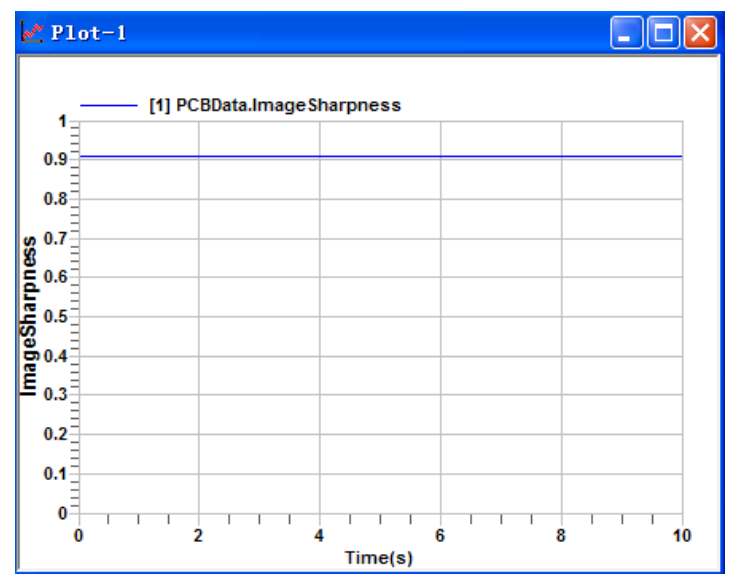

Figure. 5 Result of image sharpness value

\section{Conclusion}

This paper studies the imaging principle and the imaging process of the linear CCD imaging system of PCB AOI. And the simulation model of the entire PCB AOI imaging system is established based on the unified modeling language Modelica. It can provide some realistic guidance for the research and development of the PCB AOI systems.

\section{References}

[1] L.X. Yao, W.X. Zhang and J.L. Lian, The Application of AOI System in the PCB. Test and Measurement Technology, 2004 (112): 25-28.

[2] M.R. Sun, Application of PCB AOI Equipment in the Main Production Processes of SMT. Electronic Equipment. 2012 (01): 42-46.

[3] Y.M. Hu and Y. Tan, The Application Status and Development of AOI in China. Microcomputer Information, 2006, 22 (2-1): 143-146. 
[4] Y. Z. Wu, M.F. Wu and L. P. Chen, Research on Modelica Based Unified Modeling Language Platform of Complex Mechanical System. China Mechanical Engineering 2006 (22).

[5] Q.Y. Wang, CCD Application Technology(Tianjin University Press,China,2000).

[6] Q.S. Peng, Algorithm Based Realistic Computer Graphics. Beijing: Science Press 1999:6-26.

[7] J.J. Zhao, J.W. Ding and F.L. Zhou, Modelica Language and Unified Mechanism of Multi-domain Modeling and Simulation. System Simulation. 2006,18 (2): 570-573.

[8] J. Han and J. Liu, Engineering Optics(Defense Industry Press,China,2012).

[9] Y.B. Li, Modelica based Modeling and Simulation of Flexible Multi-body System( MS.,Huazhong University of Science and Technology, China 2011)10-19.

[10]L. Dong and T. Li, Research on CCD Imaging Model and Simulation System. System Simulation 2009, 14: 4523-4527. 